\title{
Montmorillonite as anti-tuberculosis rifampicin drug carrier - DFT and experimental study
}

\author{
E. SCHOLTZOVÁ ${ }^{1 *}$, L. JANKOVIČ ${ }^{1}$ AND D. TUNEGA ${ }^{2,3}$
}

${ }^{1}$ Inst of Inorg Chem, SAS, Dúbravská cesta 9, SK-845 36 Bratislava, Slovakia (*eva.scholtzova@savba.sk, lubos.jankovic@savba.sk)

${ }^{2}$ Univ für Bodenkultur, Peter-Jordan-Strasse 82b, A-1190

Wien, Austria (daniel.tunega@boku.ac.at)

${ }^{3}$ School of Pharmaceutical Science and Technology, Tianjin University, Tianjin, 300072, P. R. China

The intercalated montmorillonite (Mt) nanocomposites revealed a sustained release behaviour which could be utilized to design controlled release anti-tuberculosis drug carriers such as rifampicin (RIF) [1]. In these systems, the interactions of drug with smectic clay play important role in the stability of drug-carrier system.

Present study combines the use of theoretical and experimental methods to describe the interactions of the RIF intercalated in the Mt structure (RIF-Mt). DFT (Density functional theory) method with PBE functional and D3 dispersion corrections was employed for model study of RIFMt. Calculations showed, for example, that ansa chain of RIF was bent in the interlayer space of Mt in comparison with monocrystal structure of RIF. The RIF molecule was anchored to surface of the Mt layers through a plenty of hydrogen bonds mostly of a weak strength (Fig. 1).

Based on this knowledge, further experiments on the RIF intercalated to Mt modified by tetraalkylphosphonium cations (alkyl= ethyl-, butyl-, hexyl- and octyl- ) were performed to characterize RIF-modified Mt carrier systems.

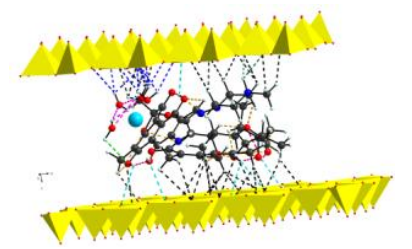

Figure 1: Hydrogen bonds in the RIF-Mt structure.

Acknowledgements: ES and LJ are grateful for the financial support by the Scientific Grant Agency VEGA (2/0021/19) and Slovak Research and Development Agency (APVV-15-0741, APVV-150347).

[1] Verma \& Riaz (2018) Int J Pol Mat and Pol Biomat 67, 221-228 